\section{EMBRYRIDDLE \\ Aeronautical University}

SCHOLARLY COMMONS
International Journal of Aviation, Aeronautics, and Aerospace

Volume 6 | Issue 4

Article 2

2019

\title{
On Atmospheric Lapse Rates
}

Nihad E. Daidzic

AAR Aerospace Consulting, LLC, aaraerospace@cs.com

Follow this and additional works at: https://commons.erau.edu/ijaaa

Part of the Atmospheric Sciences Commons, Aviation Commons, Engineering Physics Commons, and the Meteorology Commons

\section{Scholarly Commons Citation}

Daidzic, N. E. (2019). On Atmospheric Lapse Rates. International Journal of Aviation, Aeronautics, and Aerospace, 6(4). https://doi.org/10.15394/ijaaa.2019.1374

This Special Purpose Document is brought to you for free and open access by the Journals at Scholarly Commons. It has been accepted for inclusion in International Journal of Aviation, Aeronautics, and Aerospace by an authorized administrator of Scholarly Commons. For more information, please contact commons@erau.edu. 
Horizontal and vertical flow in atmosphere and cloud formation and dissipation is of fundamental importance for meteorologists, climatologists, weather forecasters, geophysicists and other related professions. The cloud coverage also regulates terrestrial albedo, which significantly regulates energy balance of the Earth. Vertical motion of air, temperature distribution and formation of clouds is also of essential importance to air transportation and flying in general. Strong updrafts, low visibility, turbulence, wind-shear and many other hazardous effects are tied to vertical motion of moist air and thus critical to pilots, dispatchers, operators, and ATC. Atmospheric lapse rate (ALR) is a central ingredient in vertical stability of air and generation of clouds with extensive vertical development. Cumulonimbus clouds (CB) are the principal generators of severe weather and most flying hazards. Low air-temperature vs. dew point (DP) depression (or spread) may cause condensation of water vapor to occur on the surface causing fog, with predictable low-visibility hazards necessitating IFR operations.

In this article, we study various atmospheric (temperature) LRs (Lapse Rates), such as, DALR (Dry air Adiabatic Lapse Rate), MALR (Moist unsaturated air Adiabatic Lapse Rate), SALR (Saturated air Adiabatic Lapse Rate), ISALR (International Standard Atmosphere Lapse Rate), and DPLR (Dew Point Lapse Rate). ELR (Environmental or actual Lapse Rate) represents actual environmental vertical air temperature distribution, which may cause vertical air temperature distribution to be unstable initiating thermals if other triggering mechanisms are also present. Air and DP temperatures often control the condensation process and the formation of cumulus clouds, which theoretically could start at the Lifting Condensation Level (LCL) or bit higher depending on other factors. For example, knowledge of atmospheric LRs is essential in glider/soaring operations where vertical air instability is welcome occurrence (Reichmann, 1993). On the other hand, flying below cumulus clouds is regarded as annoyance for airplane and helicopter flights due to associated rough rides. Ultimately, moist thermals can grow into real hazard as the cumulus clouds grow into towering ferocious CBs (Forrester, 1981; Tufty, 1987) with significant implications to aviation safety.

We can define an arbitrary vertical temperature profile for linear troposphere as:

$$
T(H) \approx T(z)=T_{0}-\Gamma_{x} \cdot z \quad z \approx H\left(1+\frac{H}{R_{0}}\right) \approx H \quad R_{0}=6371 \mathrm{~km} \quad H \leq 10 \mathrm{~km}
$$

Geopotential $(\mathrm{H})$ and orthometric $(\mathrm{z})$ heights differ little for lower terrestrial atmosphere (Daidzic, 2015a, 2015b; Iribrane and Godson, 1981). ALR $\left(\Gamma_{x}\right)$ will depend on the nature of the thermodynamic process and air properties:

$$
\Gamma_{x}=\left(\frac{d T}{d H}\right)_{x} \approx\left(\frac{d T}{d z}\right)_{x} \leq \geq 0
$$

Constant lapse-rate processes occur in atmospheric layers if the air is being lifted adiabatically (often meant isentropically) where large mass of upward moving air is behaving as a closed material system not exchanging mass or energy with the surrounding. This is an essential concept in atmospheric thermodynamics. We will also explore dry- and wet-adiabats, which serve important role in construction of many thermodynamic diagrams (such as "Skew-T vs. Log-p") 
used extensively in meteorology, weather predictions, and soaring operations. DP is the temperature at which first condensation event occurs or the temperature achieved by cooling moist air at constant pressure (isobaric) and constant specific humidity or mixture ratio (isohumes) at which water vapor becomes fully saturated (in thermodynamic equilibrium with the plane liquid surface). That does not automatically imply clouds will invariably form, but merely it provides required ingredients for formation. Similarly, the frost point (FP) is the temperature to which air must be cooled isobarically to achieve equilibrium with the plane surface of pure solid-water (ice).

The concept of ALRs and LCLs is mentioned in more or less detail in some aviation- and pilot-oriented books dealing with the basics of aviation weather and weather flying, such as, Bradbury (2000), Cosgrove (1999), FAA (2016), Jeppesen (2015), Lester (2007), Piggot (1996), and Reichmann (1993). Understandably, these practical books on aviation and flying weather lack serious treatment of atmospheric thermodynamics and/or providing deeper insights into ALR and LCL although it must be pointed out that Bradbury's analysis goes deeper into the subject matter than others.

This article serves as a review of ALRs, but it also introduces the analysis and effects of gravitational acceleration, deeper SALR analysis, and small perturbations analysis with respect to gravitational and thermodynamic parameters. We contribute a sensitivity analysis of various ALRs, something that has never been discussed previously to the best of our knowledge.

\section{Mathematical models of lapse rates}

Theoretical models of DALR, MALR, SALR, and DPLR are derived. ISALR is a standard and is mentioned for completeness and comparison. More information on terrestrial standard atmosphere and various constant and variable LRs can be found in Daidzic (2015a, 2015b).

\section{Dry Air Adiabatic Lapse Rate}

The First Law-of-Thermodynamics for closed material system in two different differential forms per unit mass using internal energy $u$ and thermodynamic work and using enthalpy $h=u+p v$ and technical work yield:

$$
\delta q=d u+p d v=c_{v} d T+p d v \quad \text { or } \quad \delta q=d h-v d p=c_{p} d T-v d p
$$

In this form, heat and work are not exact differentials and do not present states of the system. Assuming reversibility, the first Law-of-Thermodynamics via entropy formulation yields:

$$
d s=\frac{\delta q}{T}=c_{v d} \frac{d T}{T}+\frac{p}{T} d v \quad \text { and } \quad d s=\frac{\delta q}{T}=c_{p d} \frac{d T}{T}-\frac{v}{T} d p
$$

If the process is isentropic (reversible), then there is also no heat exchange (adiabatic) and entropy does not change as the air parcel is lifted vertically:

$$
d s=c_{p d} \frac{d T}{T}-\frac{v}{T} d p=0
$$


By utilizing the ideal-gas law and rearranging, we obtain:

$$
\frac{d T}{T}-\left(\frac{R_{d}}{c_{p d}}\right) \frac{d p}{p}=0 \Rightarrow d(\ln T)=\left(\frac{\gamma_{d}-1}{\gamma_{d}}\right) d(\ln p)
$$

Real-gas effects cause specific heat capacity of air at constant pressure to be a weak function of temperature. We now employ the aerostatic (hydrostatic) equation, which links atmospheric air pressure changes with the height (altitude) in still and well mixed atmosphere. Classical hydrostatic/aerostatic equation neglects terrestrial sphericity and change in gravitational acceleration with height (Daidzic, 2015a, 2015b):

$$
\frac{\partial p}{\partial z}=-\rho(z) \cdot g_{0} \quad g_{0} \approx 9.80665 \mathrm{~m} / \mathrm{s}^{2}=32.174 \mathrm{ft} / \mathrm{s}^{2}
$$

Combining the ideal-gas law and the hydrostatic equation, we obtain from Eqs. (6) and (7):

$$
\frac{d T}{T}-\left(\frac{R_{d}}{c_{p d}}\right) \frac{g_{0} d z}{p} \cdot \rho=0 \quad T>0
$$

Simplifying and reducing Eq. (8), we obtain:

$\operatorname{DALR}=\Gamma_{d}=\left(-\frac{d T}{d z}\right)_{d}=\frac{g_{0}}{c_{p d}}$

Hence for isentropic process, the ALR of non-condensable air mixture is a pseudoconstant. A schematic of isentropic (including adiabatic) lifting of an air parcel is shown in Fig. 1. Taking values of air's constant-pressure specific heat capacity at $300 \mathrm{~K}\left(27^{\circ} \mathrm{C}\right)$ and the standard terrestrial gravitational acceleration, we obtain:

$$
\Gamma_{d}=\frac{g_{0}}{c_{p d}}=\frac{9.80665}{1005.7} \approx 0.00975 \frac{{ }^{\circ} \mathrm{C} \text { or K }}{\mathrm{m}}=9.75 \frac{{ }^{\circ} \mathrm{C} \text { or K }}{\mathrm{km}}=2.97 \frac{{ }^{\circ} \mathrm{C} \text { or K }}{1,000 \mathrm{ft}}
$$

Specific heat capacities at constant pressure and at constant volume of dry air are weak functions of temperature (real-gas effects), but in the normal range of terrestrial atmospheric temperatures we can often assume it to be constant. Additionally, terrestrial gravitational acceleration is not constant and is normally larger in polar- than in equatorial-regions (apart from gravitational anomalies). Additionally, the gravitational acceleration decreases slightly with height (Daidzic, 2015a, 2015b). Simultaneously, Earth is spherical/ellipsoidal, which introduces another small correction in the flat-Earth hydrostatic/aerostatic equation. For example, Ambaum (2008) discusses hydrostatic relationship for curved surfaces and computes geometric reduction in surface pressure as large as 5 mbar on Earth and 39 mbar on Titan (moon of Saturn). Berberan-Santos et al. (1997) derived and discussed barometric formula for non-uniform gravitational fields. The 
international gravity formula (IGF) represents the current model of Earth's gravitation, which includes the rotation of the Earth and geodetic (GD) latitude (Stacey and Davis, 2008):

$g\left(\varphi_{G D}\right)=9.780327 \cdot\left[1+0.0053024 \cdot \sin ^{2}\left(\varphi_{G D}\right)+0.0000059 \cdot \sin ^{2}\left(2 \varphi_{G D}\right)\right]$

This is the reference variation of terrestrial gravity on the surface and any aberration from it is referred to as gravitational anomaly (Stacey and Davis, 2008). Gravitational effects are treated in Iribarne and Godson (1981), Lowrie (2007), Stacey and Davis (2008), Torge (2001).

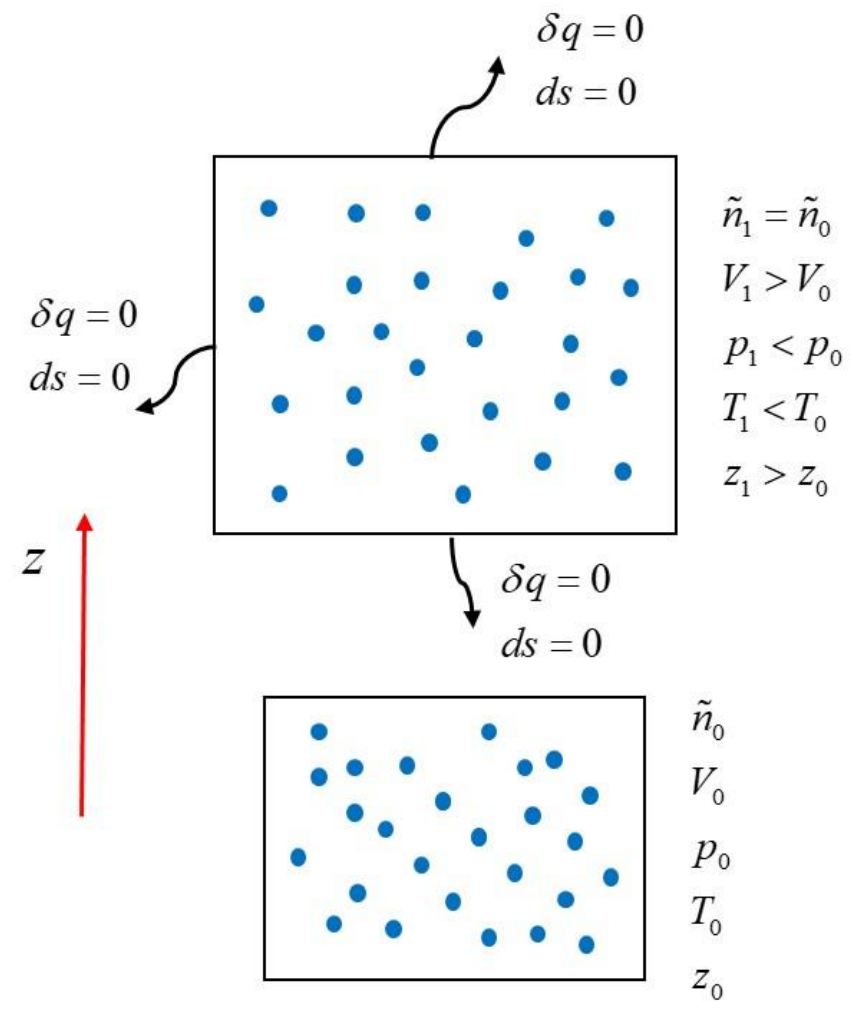

Figure 1: Isentropic lifting of an air parcel.

In the analysis of the standard atmosphere (ISA) it is assumed that Earth is a perfect sphere of uniform mass density (Daidzic, 2015a). The gravitational equipotential surfaces are concentric spheres with the gravitational acceleration vectors being equivalent to the radius vectors emanating from the geocenter (geometric center) and barycenter (mass center). Hence, the gravitational acceleration changes only as a function of altitude above the Geoid, which below $500 \mathrm{~km}$ or $1,640,000 \mathrm{ft}$ (Daidzic, 2015a) is linearized to:

$g=g_{o}\left(\frac{R_{0}}{R_{0}+z}\right)^{2} \approx g_{o}\left(1-2 \frac{z}{R_{0}}\right) \quad g_{o}=9.80665 \mathrm{~m} / \mathrm{s}^{2}=32.174 \mathrm{ft} / \mathrm{s}^{2}$

For example, Iribarne and Godson (1981) present DALR and other atmospheric LRs in geopotential (GP) height units. Daidzic (2015a, 2015b) derives relationships between orthometric 
(z) and geopotential $(\mathrm{H})$ heights and recalculates ISA to about $86 \mathrm{~km}$ in both heights. Thus, Eq. (8) is better stated in terms of GP heights. However, in lower atmosphere, differences between the GP and orthometric heights are small and can often be neglected (Daidzic, 2015a).

Dry air is calorically non-perfect gas. To estimate the sensitivity of DALR to small changes in the constant-pressure specific heat capacity and/or gravitational acceleration changes, we perform linear small-perturbation analysis:

$$
\Delta \Gamma_{d}=\left(\frac{\partial \Gamma_{d}}{\partial g}\right)_{c_{p d}} \Delta g+\left(\frac{\partial \Gamma_{d}}{\partial c_{p d}}\right)_{g} \Delta c_{p d} \quad\left(\frac{\partial \Gamma_{d}}{\partial g}\right)_{c_{p d}}=\left(\frac{1}{c_{p d}}\right)_{0} \quad\left(\frac{\partial \Gamma_{d}}{\partial c_{p}}\right)_{g}=-\left(\frac{g_{0}}{c_{p d}^{2}}\right)_{0}
$$

Relative changes of DALR due to small relative changes in gravitational acceleration and/or specific heat capacity at constant-pressure are:

$$
\frac{\Delta \Gamma_{d}}{\Gamma_{d}}=\left(\frac{1}{g_{0}}\right) \Delta g-\left(\frac{1}{c_{p d}}\right)_{0} \Delta c_{p d}
$$

Accordingly, a $1 \%$ change in gravitational acceleration, due to height, latitude and/or gravitational anomaly changes would result in a $1 \%$ proportional change in DALR (about $0.03^{\circ} \mathrm{C}$ ) and hence negligible for most terrestrial applications. However, this may be a significant effect on other planets and satellites with respective atmospheres (e.g. Mars, Venus, etc.). Generally, meteorological temperature measurements are made with an accuracy of $0.05^{\circ} \mathrm{C}$ to enable rounding to one decimal place. A $1 \%$ increase in $c_{p d}$ would result in a $1 \%$ decrease in DALR. High-latitude regions ( $\mathrm{S}$ and $\mathrm{N}$ ) experience increased local gravitational accelerations (due to flattened poles, being closer to the center, and decrease in centrifugal force from Earth's own angular rotation). Simultaneously, colder climates result in overall slightly lower specific heat capacities of air. Both effects lead to increasing DALR at higher latitudes. On the other hand, tropical regions experience lowering gravitational accelerations. Simultaneously, air has somewhat higher specific heat capacities due to higher average temperatures, both effects leading to slightly reduced DALR. Overall, the effect on DALR perturbations is small. It is common practice to round it and just use DALR of $10^{\circ} \mathrm{C}$ (or K) per each $1,000 \mathrm{~m}(1 \mathrm{~km}), 1^{\circ} \mathrm{C} / 100 \mathrm{~m}$, or $3^{\circ} \mathrm{C}$ per $1,000 \mathrm{ft}$.

\section{Moist Air Adiabatic Lapse Rate}

As moist (or humid) unsaturated air is lifted vertically up, it cools due to isentropic expansion similarly as dry air, but at slightly lower rates (Dutton, 2002; Rogers, 1979; Saucier, 1989). Only in very low temperature regions and/or arid conditions, is the amount of water vapor so low that air lifting adiabatically cools practically as DALR. Unsaturated moist air is generally cooling at MALR. Difference exists due to constant-pressure specific heat capacity of water vapor $(1,867 \mathrm{~J} / \mathrm{kg} \mathrm{K})$ being about $85 \%$ higher than of dry air mixture (about 1,006 J/kg K). Since the amount of water vapor is small (typically 5-20\%o mass fraction), the MALR is only slightly lower than DALR. Since there is practically no release of latent heat during adiabatic ascent of moist air, we utilize the same analysis used for DALR derivation previously: 
$\operatorname{MALR}=\Gamma_{M}=\left(-\frac{d T}{d z}\right)_{M}=\frac{g_{0}}{c_{p m}} \quad z \approx H$

The specific heat capacity at constant pressure for moist air as a function of specific humidity was derived in Daidzic (2019). Accordingly, MALRs are variable, all slightly dependent on the specific humidity (or mixture ratios) at the reference surface:

$$
\Gamma_{M}=\frac{g_{0}}{c_{p m}} \approx \frac{g_{0}}{c_{p d} \cdot(1+0.856 \cdot \mathrm{q})}=\Gamma_{d}(1-0.856 \cdot \mathrm{q})<\Gamma_{d}
$$

MALR is also a pseudo-constant as the specific humidity remains constant during isentropic/adiabatic lifting, although $\mathrm{RH}$ increases. For example, for moist air with specific humidity of 0.02 ( $\mathrm{kg}$ water vapor/kg dry air), MALR is:

$$
\Gamma_{M}=2.97 \cdot(1-0.856 \cdot 0.02) \approx 2.92 \mathrm{~K} / 1000 \mathrm{ft}=9.58 \mathrm{~K} / \mathrm{km}
$$

Hence, in this case MALR is about $1.7 \%$ lower than DALR. In very warm and humid climates, MALR may be 3-4\% lower than DALR. Due to infinite variations in surface-air humidity, DALR is used as a boundary defining absolute vertical instability of air parcels. Measured ELR larger than DALR (in absolute values) imply vertically (statically) unstable air. On the other hand, isothermal and inversion layers are absolute stable with lighter air on top of the heavier air (Rayleigh-Taylor stability problem). Atmospheric lapse rates and stability is treated in many specialized references, such as, Dutton (2002), Iribarne and Cho (1980), Iribarne and Godson (1981), Rogers (1979), Saucier (1989), Stull (2016), Tsonis (2007), Wallace and Hobbs (2006).

\section{Saturated Air Adiabatic Lapse Rate}

Once moist aid is fully saturated at DP or $100 \% \mathrm{RH}$, the rate of cooling will slow down due to condensation of excess of water vapor and release of the latent-heat of evaporationcondensation, which then reduces expansion cooling of air parcels. In reality, some supersaturation must exist in atmosphere and the water vapor will not start condensing at exactly $100 \%$ $\mathrm{RH}$, but few percentages above due to heterogeneous nucleation in the abundant presence of condensation nuclei $(\mathrm{CN})$. Definition of saturation vapor pressure assumes equilibrium in condensation-evaporation rates over plane liquid surfaces, while the nucleation sites in atmosphere have curvatures and thus require higher vapor pressures due to surface energy (Laplace law). The smaller the droplets, the higher the super-saturation is required (Fleagle and Businger, 1980). On the other extreme, homogeneous nucleation takes place in very clean air free of tiny suspended aerosols and/or small solid particles, but requires super-saturations of few hundred percentages, which is not easily found in lower atmosphere.

Estimating SALR (Saturated air Adiabatic Lapse Rate) is more difficult as it involves phase change of condensable air component, i.e., water, in the presence of inert dry-air mixture. As unsaturated air ascends, it also cools down until it reaches DP. Strictly, DP is defined as a temperature at which water-vapor saturation (or molecular equilibrium between the vapor-liquid condensation-evaporation rates) is just reached under constant pressure (isobaric), while also 
maintaining constant mixture ratio (or specific humidity) until condensation occurs. Any further cooling will be followed by condensation of excess water vapor. DP is not identical to wet-bulb (WB) temperature. Psychrometer's WB temperature, or more relevant thermodynamic wet-bulb temperature, is achieved during adiabatic saturation and follows constant enthalpy lines (isenthalpic) and is larger than DP. Only at $100 \% \mathrm{RH}$ are DP, WB, and dry-bulb (DB) temperatures identical. During condensation, some water vapor transforms into water droplets (typically 20-50 micrometers cloud droplets). When saturated air $(100 \% \mathrm{RH})$ is lifted, the adiabatic cooling process continues under variable SALR.

Typically, in SALR models and computations it is assumed that condensed water (droplets) precipitate defining the, so-called, pseudo-adiabatic process (Dutton, 2002; Houghton, 2002; Iribarne and Cho, 1980; Iribarne and Godson, 1981; Rogers, 1979; Saucier, 1989; Tsonis, 2007). A recommendation proposed by the International Meteorological Organization (IMO) in 1947 and used by Saucier (1989) and Iribarne and Godson (1981), yields:

$$
\left(c_{p}+c_{w} \cdot r\right) \frac{d T}{T}-R_{d} \frac{d p}{p}+d\left(\frac{l_{v} \cdot r}{T}\right)=0 \quad l_{v}=\frac{L_{v}}{m} \quad c_{p} \gg c_{w} \cdot r
$$

According to Dutton (2002), Iribarne and Cho (1980), Iribarne and Godson (1981), Rogers (1979), Tsonis (2007), and Wallace and Hobbs (2006), the first Law-of-Thermodynamics applied to an open material system in which temperature-independent latent-heat of condensation is released while condensed liquid droplets precipitate, defining pseudo-adiabats. That process results in the following differential equation:

$c_{p} \frac{d T}{T}-R_{d} \frac{d p}{p}=-\frac{l_{v} \cdot d r}{T} \quad l_{v}=l_{\mathrm{lg}}$

The Clapyeron and the Clausius-Clapeyron equations are used to define equilibrium states during phase transitions (vaporization, fusion, sublimation). Derivation of both equations and some solutions for various phase transitions are given in Daidzic (2019). During ascent of saturated air, the mixing ratio (specific humidity) remain at their respective saturation values $\left(r=r_{s}\right.$ and $\left.\sigma=\sigma_{s}\right)$. The differential amount of condensing water vapor is:

$$
d r_{s}=0.622 \frac{e_{s}}{p}\left(\frac{d e_{s}}{e_{s}}-\frac{d p}{p}\right)
$$

By applying Clausius-Clapeyron equation on the relative differential amount of condensate:

$$
\frac{d r_{s}}{r_{s}}=\frac{d e_{s}}{e_{s}}-\frac{d p}{p}=\frac{l_{v}}{R_{v}} \frac{d T}{T^{2}}-\frac{d p}{p}
$$

Substituting into Eq. (17), yields: 
$c_{p} \frac{d T}{T}-R_{d} \frac{d p}{p}=\frac{l_{v} r_{s}}{T} \frac{d p}{p}-\frac{l_{v}^{2} r_{s}}{R_{v} T^{2}} \frac{d T}{T}$

By rearranging variables:

$\left(c_{p}+\frac{l_{v}^{2} r_{s}}{R_{v} T^{2}}\right) \frac{d T}{T}-\left(R_{d}+\frac{l_{v} r_{s}}{T}\right) \frac{d p}{p}=0$

A differential form of Hypsometric equation (ideal-gas plus hydrostatic equation) for air yields:

$\frac{d p}{p}=-\frac{\rho_{m} g_{0} d z}{p}=-\frac{\rho_{m} g_{0}}{\rho_{m} R_{m} T} d z=-\frac{g_{0}}{R_{m} T} d z$

By substituting Eq. (22) into Eq (20) and reducing, one obtains:

$$
\left(c_{p}+\frac{l_{v}^{2} r_{s}}{R_{v} T^{2}}\right) \frac{d T}{T}=-\left(1+\frac{l_{v} r_{s}}{R_{d} T}\right) \frac{g_{0}}{T} d z
$$

We approximated the moist-air gas-constant (Daidzic, 2019) with the one for dry air since the difference is negligible compared to magnitude of the latent-heat. Since maximum mixture ratios are approximately $10^{-2}\left(\mathrm{~kg} \mathrm{H}_{2} \mathrm{O} / \mathrm{kg}\right.$ dry air), we obtain:

$\left(-\frac{d T}{d z}\right)_{s} \cdot\left(1+\frac{l_{v}^{2} r_{s}}{c_{p} R_{v} T^{2}}\right)=\left(\frac{g_{0}}{c_{p}}\right) \cdot\left(1+\frac{l_{v} r_{s}}{R_{d} T}\right)$

The moist air gas constant is only very slightly higher than of dry air. Finally, we have:

$\Gamma_{s}=S A L R=\left(-\frac{d T}{d z}\right)_{s}=\Gamma_{d} \cdot\left(1+\frac{l_{v} r_{s}}{R_{d} T}\right) \cdot\left(1+\frac{l_{v}^{2} r_{s}}{c_{p} R_{v} T^{2}}\right)^{-1}=\Gamma_{d} \cdot \phi\left[r_{s}(T)\right]$

Where SALR-function is,

$\phi(T)=\frac{S A L R}{D A L R}=\left(1+\frac{l_{v} r_{s}}{R_{d} T}\right) \cdot\left(1+\frac{l_{v}^{2} r_{s}}{c_{p} R_{v} T^{2}}\right)^{-1} \leq 1$

It is interesting to evaluate the first and the second derivatives of the SALR-function. We can directly differentiate Eq. (26) with respect to absolute temperature. However, saturation mixture ratio is also function of temperature as will be seen later utilizing the Clapeyron-Clausius (C-C) equation. Additionally, the resulting expressions for derivatives are extremely complex and were checked using MAPLE ${ }^{\mathrm{TM}}$ symbolic computations software. Hence, the analytical analysis and numerical root-finding of resulting polynomial will not be shown here. Instead, we use second- 
order central-difference numerical differentiation formulas to evaluate $1^{\text {st }}$ and $2^{\text {nd }}$ derivatives of SALR-function as a function of temperature:

$\frac{d \phi\left(T_{i}\right)}{d T} \approx \frac{\phi\left(T_{i+1}\right)-\phi\left(T_{i-1}\right)}{2 \cdot(\Delta T)}+O\left[(\Delta T)^{2}\right]$

and

$\frac{d^{2} \phi\left(T_{i}\right)}{d T^{2}} \approx \frac{\phi\left(T_{i+1}\right)-2 \cdot \phi\left(T_{i}\right)+\phi\left(T_{i-1}\right)}{(\Delta T)^{2}}+O\left[(\Delta T)^{2}\right]$

As it will be seen later, SALR has inflection point around $+9^{\circ} \mathrm{C}$ at normal SL atmospheric pressure. The rate of SALR change first increases (as negative value) and then after inflexion point starts decreasing approaching lower asymptote. This result was never mentioned before to the best of our knowledge. We can further simplify this equation under the assumption that latent heat of vaporization, specific heat capacity at constant pressure, and other thermodynamic parameters are constant. Following Dutton (2002), we linearize Eq. (26) around $273 \mathrm{~K}\left(0^{\circ} \mathrm{C}\right)$ :

$$
\frac{l_{v}}{R_{d} T}=\frac{2.5 \times 10^{6}}{287 \times 273} \approx 32 \quad \frac{l_{v}^{2}}{c_{p} R_{v} T^{2}}=\frac{\left(2.5 \times 10^{6}\right)^{2}}{1005 \times 461.5 \times(273)^{2}} \approx 180
$$

and:

$$
\phi(T) \approx \frac{1+32 \cdot r_{s}}{1+180 \cdot r_{s}}<1
$$

For example, saturated-air mixture ratio at $288 \mathrm{~K}\left(15^{\circ} \mathrm{C}\right)$ and $\mathrm{SL}$ atmospheric pressure can be used from the equation derived in Daidzic (2019) as:

$$
r_{s} \approx 0.622 \frac{e_{s}}{p}=0.622 \frac{611.657}{p} \cdot \exp \left(19.83-\frac{5417}{T}\right) \approx 1.1 \times 10^{-2}=\mathrm{O}\left(10^{-2}\right)
$$

Using Eq. (26), gives SALR function of $\phi(T) \approx 0.450$ and SALR in this particular case yields:

$$
\Gamma_{s}=\Gamma_{d} \cdot \phi(T) \approx 1.34[\mathrm{~K} / 1000 \mathrm{ft}] \approx 4.38[\mathrm{~K} / \mathrm{km}]
$$

For arbitrary temperatures, we need to utilize SALR described with Eqs. (25) and (26). The SALR is typically in the range $6-7 \mathrm{~K} / \mathrm{km}$, but can reach $5 \mathrm{~K} / \mathrm{km}$ in warm humid climates and as high as $9 \mathrm{~K} / \mathrm{km}$ in cold dry areas. Hence, average SALR is roughly two-thirds of DALR, which is about the same as tropospheric ISA LR. The same arguments we used for DALR's small dependence on gravitational acceleration and temperatures applies to SALR, but that analysis is not shown.

Dew Point Lapse Rate 
We will now derive Dew Point Lapse Rate (DPLR). DP defines temperature at which cooled air (diabatic isobaric process) reaches vapor-liquid equilibrium at constant pressure and constant specific humidity (isohumes) and is defined by a horizontal line in the $p$ - $T$ diagram (Daidzic, 2019a). As air parcel lifts, it experiences reduced atmospheric pressures and cools down by doing thermodynamic work of expansion. No actual condensation and removal of condensate takes place as in SALR. The saturation mixture ratio remains constant during adiabatic lifting:

$$
d r_{s}=0.622 \frac{e_{s}}{p}\left(\frac{d e_{s}}{e_{s}}-\frac{d p}{p}\right)=0 \Rightarrow \frac{d e_{s}}{e_{s}}=\frac{d p}{p}
$$

The partial pressure of water vapor at saturation obeys the Clausius-Clapeyron equation:

$e\left(T_{D P}\right)=e_{s}\left(T_{D P}\right) \quad \frac{d e_{s}}{d z}=\frac{d}{d z}\left[e_{s}\left(T_{D P}\right)\right]=\frac{d e_{s}}{d T_{D P}} \frac{d T_{D P}}{d z}$

The relationship between water vapor and air pressure is:

$$
e=\left(\frac{r}{r+\varepsilon}\right) p \Rightarrow \frac{d e}{d z}=\left(\frac{r}{r+\varepsilon}\right) \frac{d p}{d z}=\left(\frac{e}{p}\right) \frac{d p}{d z}
$$

The rate at which the saturation pressure decreases with altitude is much slower than the atmospheric pressure rate reduction, but the relative change of both pressures is the same:

$$
\frac{1}{e} \frac{d e}{d z}=\frac{1}{p} \frac{d p}{d z} \quad r=\text { const. }
$$

As the saturation vapor pressure decreases so does DP:

$$
\frac{1}{p} \frac{d p}{d z}=-\frac{1}{e_{s}} \frac{d e_{s}}{d T_{D P}} \Gamma_{D P} \quad \Gamma_{D P}=-\frac{d T_{D P}}{d z}
$$

The $C$ - $C$-equation for (water-vapor) saturation vapor pressure yields:

$$
d\left(\ln e_{s}\right)=\frac{d e_{s}}{e_{s}}=\frac{l_{v}}{R_{v}} \cdot \frac{d T_{D P}}{T_{D P}^{2}}
$$

As the lifting process is adiabatic, the $p-T$ relationship for moist air produces:

$$
\frac{d T}{T}-\frac{R_{m}}{c_{p m}} \frac{d p}{p}=0 \Rightarrow \frac{1}{T} \frac{d T}{d z}=\left(\frac{\gamma_{m}-1}{\gamma_{m}}\right) \frac{1}{p} \frac{d p}{d z}
$$

By using MALR definition from Eq. (15), we obtain: 
$\frac{1}{p} \frac{d p}{d z}=-\frac{\Gamma_{d}(1-0.86 \cdot \mathrm{q})}{T}\left(\frac{\gamma_{d}}{\gamma_{d}-1}\right)(1+0.25 \cdot \mathrm{q})=-\frac{1}{e_{s}} \frac{d e_{s}}{d T_{D P}} \Gamma_{D P}=-\frac{l_{v}}{R_{v} \cdot T_{D P}^{2}} \Gamma_{D P}$

By neglecting $2^{\text {nd }}$-order terms in specific humidity, DPLR becomes:

$$
\Gamma_{D P}=\operatorname{DPLR}=\left(-\frac{d T}{d z}\right)_{D P}=\Gamma_{M}\left(\frac{\gamma_{m}}{\gamma_{m}-1}\right) \frac{R_{v}}{l_{v}} \frac{T_{D P}^{2}}{T} \approx \Gamma_{d}\left(\frac{\gamma_{d}}{\gamma_{d}-1}\right)(1-0.61 \cdot \mathrm{q})\left(\frac{R_{v}}{l_{v}}\right)\left(\frac{T_{D P}^{2}}{T}\right)
$$

Introducing DP depression (spread):

$D=T-T_{D P} \geq 0$

DPLR becomes:

$$
\begin{aligned}
& \Gamma_{D P}=\Gamma_{d}\left(\frac{\gamma_{d}}{\gamma_{d}-1}\right)(1-0.61 \cdot \mathrm{q})\left(\frac{R_{v} T}{l_{v}}\right)\left(\frac{T_{D P}}{T}\right)^{2}=\left(\frac{R_{v}}{l_{v}}\right) \frac{\Gamma_{d} \cdot \gamma_{d}(1-0.61 \cdot \mathrm{q})}{\gamma_{d}-1} \cdot T \cdot\left(1-\frac{D}{T}\right)^{2} \approx \\
& \approx 6.299 \times 10^{-3} \cdot(1-0.61 \cdot \mathrm{q}) \cdot T \cdot\left(1-\frac{D}{T}\right)^{2}\left[\frac{\mathrm{K}}{\mathrm{km}}\right]
\end{aligned}
$$

Specific humidity $\mathbf{q}$ depends on measured air temperature and DP-depression and is not an independent variable. Using definition of RH from Daidzic (2019), we derive an expression for the surface specific humidity, which is weak function of atmospheric pressure (Daidzic, 2019):

$$
\mathrm{q}(T, D, p)=\varphi \cdot \mathrm{q}_{s}(p, T) \approx \frac{380.14}{p} \cdot \exp \left[54.015-\frac{6829.36}{T}-5.1723 \cdot \ln T-\frac{5417.12 \cdot D}{T(T-D)}\right]
$$

For example, DPLR of moist air at temperature of $20^{\circ} \mathrm{C}(293 \mathrm{~K})$ and dew point of $12^{\circ} \mathrm{C}$ $(285 \mathrm{~K})$ has RH of approximately $60 \%$. DP-depression $D$ is $8^{\circ} \mathrm{C}(8 \mathrm{~K})$. Using Eq. (38), while neglecting specific humidity contribution, DPLR yields about $0.546 \mathrm{~K} / 1,000 \mathrm{ft}(1.8 \mathrm{~K} / \mathrm{km})$. This is valid result as measured DPLRs are normally in the range $1.6-2.0 \mathrm{~K} / \mathrm{km}$ or 0.50 to $0.6 \mathrm{~K} / 1,000$ $\mathrm{ft}$. Once air temperature and DP are specified, water saturation vapor pressure, $\mathrm{RH}$ and specific humidity are all functions of it. Hence, DPLR is a function of both air temperature and DP and it is not a simple pseudo-constant as DALR. To estimate changes in DPLR as a function of small changes in DP and air temperature, we again employ small perturbations analysis by writing:

$$
\left(\frac{\partial \Gamma_{D P}}{\partial T}\right)_{T_{D P}}=-\Gamma_{M}\left(\frac{\gamma_{m}}{\gamma_{m}-1}\right) \frac{R_{v}}{l_{v}}\left(\frac{T_{D P}}{T}\right)^{2} \quad\left(\frac{\partial \Gamma_{D P}}{\partial T_{D P}}\right)_{T}=2 \Gamma_{M}\left(\frac{\gamma_{m}}{\gamma_{m}-1}\right) \frac{R_{v}}{l_{v}} \frac{T_{D P}}{T}
$$

Accordingly, the relative change about the reference point " 0 " is: 


$$
\frac{\Delta \Gamma_{D P}}{\Gamma_{D P}}=-\left(\frac{1}{T}\right)_{0} \Delta T+\left(\frac{2}{T_{D P}}\right)_{0} \Delta T_{D P}
$$

Therefore, a 5\% decrease in air temperature (or DP-depression) causes proportional 5\% increase in DPLR at constant DP, while 5\% increase of DP causes 10\% increase of DPLR at constant temperature or DP depression. In general, DALR > MALR > SALR > DPLR.

\section{ISA Troposphere Lapse Rate}

The concept of ISA (International Standard Atmosphere) troposphere uses constant lapse rate (LR) of about negative $2^{\circ} \mathrm{C}$ per $1000 \mathrm{ft}(6.5 \mathrm{~K} / \mathrm{km}$ to be more accurate). Hence, virtual ISA is conditionally stable (between DALR/MALR and SALR). More details on ISA and various thermal layers with associated lapse rates are to be found in Daidzic (2015a, 2015b), ISO (1975), ICAO (1993), and NOAA (1976). A graphic summary of various lapse rates is depicted in Fig. 2.

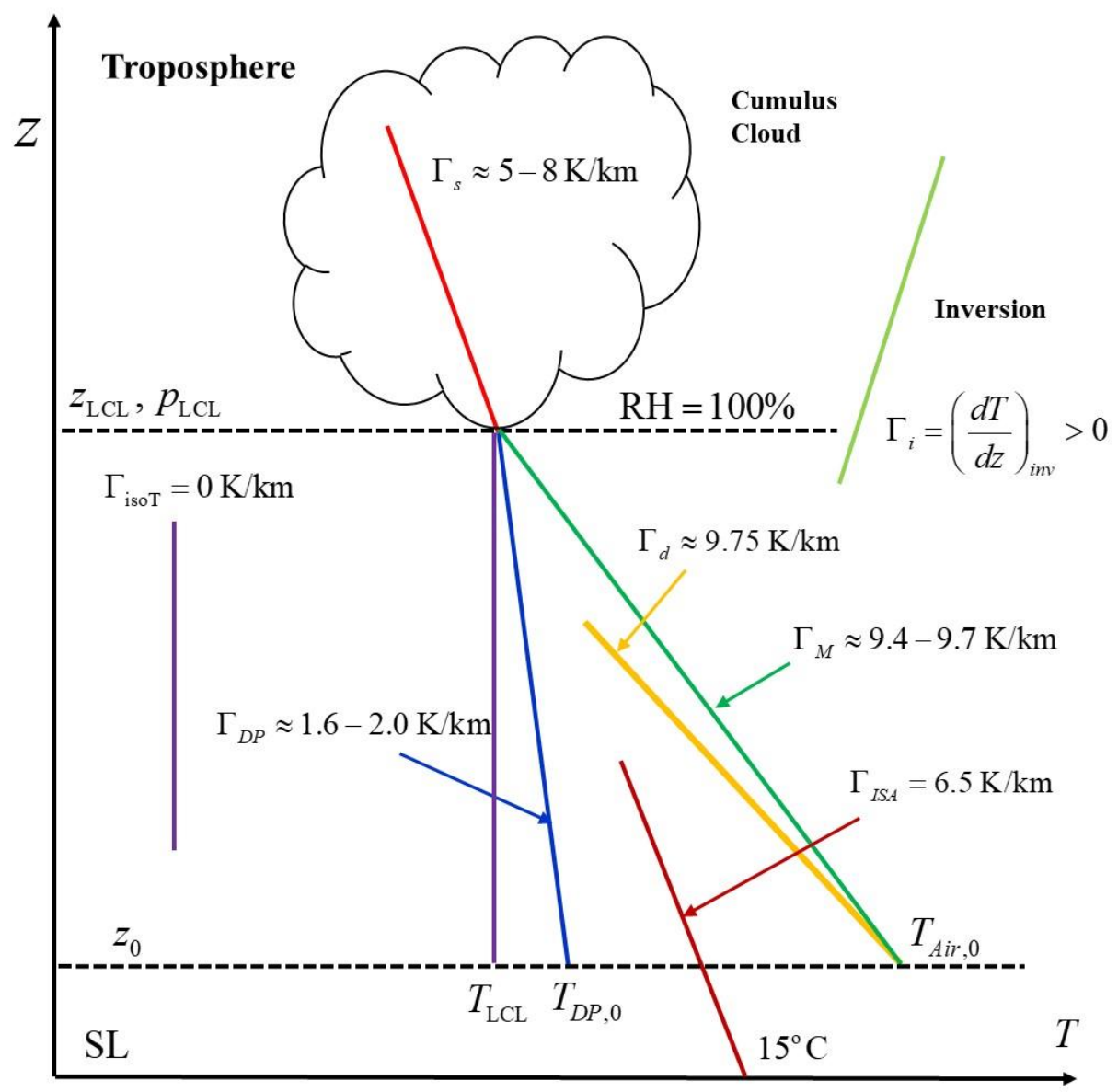

Figure 2: Graphic representation of various atmospheric LRs. ISA LR refers to ISA troposphere only. (Not to scale).

ISA tropospheric pressure is a function of temperature (Daidzic, 2015a, 2015b) where height is in geopotential linear dimension: 


$$
\begin{aligned}
& \delta=\theta^{-\lambda_{0}} \quad \sigma=\theta^{-\lambda_{0}-1} \quad \sigma=\frac{\rho\left[\mathrm{kg} / \mathrm{m}^{3}\right]}{1.225} \quad \delta=\frac{p[\mathrm{~Pa}]}{101,325} \quad \theta=\frac{T[\mathrm{~K}]}{273.15} \\
& \lambda_{0}=-\frac{g_{0}}{R \cdot \Gamma_{\mathrm{ISA}}}=-5.255823 \quad \Gamma_{\mathrm{ISA}}=\left(-\frac{d T}{d H}\right)_{\mathrm{ISA}}=6.5 \mathrm{~K} / \mathrm{gpkm} \approx 1.98 \mathrm{~K} / 1,000 \mathrm{ft}
\end{aligned}
$$

\section{Results and Discussion}

The result for SALR computations as a function of temperatures in the range of $-60^{\circ}$ to $+50^{\circ} \mathrm{C}$ are shown in Fig. 3. SALR implies wet-adiabatic lifting of saturated moist air (100\% RH). Naturally, SALR decreases at higher air temperatures as saturated warmer air has higher moisture content (higher vapor pressures), which then results in reduced expansion cooling as the air parcel is lifted up. Computed first and second derivatives of SALR function are shown in Fig. 4. The inflexion point (steepest decrease in SALR) is around $9^{\circ} \mathrm{C}$ as was mentioned before. The SALR function monotonically decreases between upper (DALR) and lower asymptote.

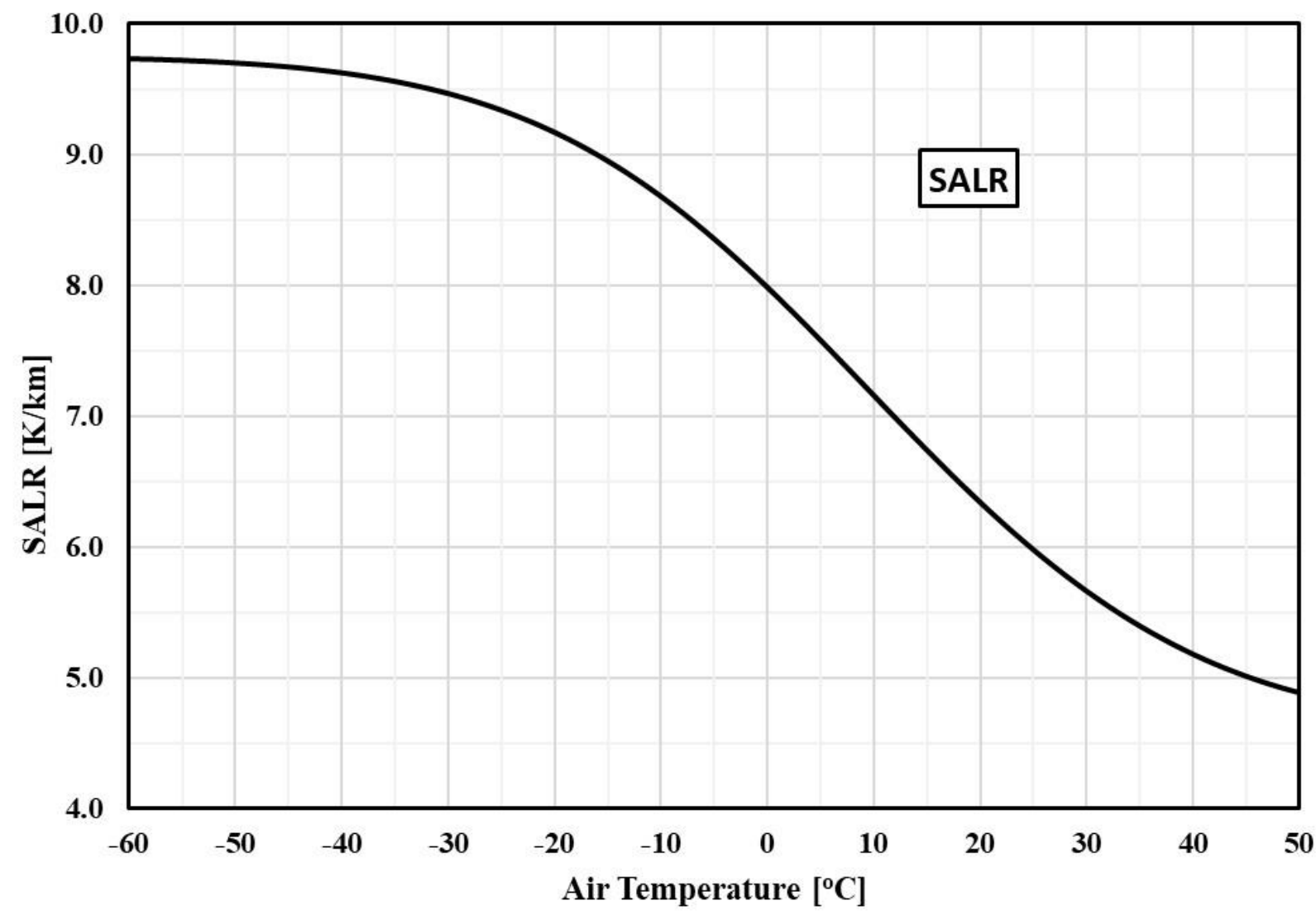

Figure 3: SALR as a function of air temperature $(\mathrm{RH}=100 \%)$.

The results of DPLR computations for various DP-depressions as parametric curves is shown in Fig. 5. As air temperature increases so does DPLR. On the other hand, as DP-depressions increase for constant air temperatures (DPs decrease), DPLRs decrease as well. There is less moisture in moist air with increased DP-depressions and lower DPs. Increasing DPLRs with air 
temperature for constant DP-depressions means that DPs are also increasing implying more moisture and lower cooling rates as water vapor has higher heat capacity than dry air. From Eqs. (38) and (40), we see that DPLR depends quadratically on DPs and inversely to air temperatures. We now consider DPLR as a function of DP-depressions and with constant air temperature parametric curves as presented in Fig. 6. As DP-depressions increase at constant air temperature, means that DP temperatures also decrease implying less moisture content in air. DPLRs will hence decrease with increasing DP-depressions for constant air temperature parametric curves. For constant DP-depressions, increasing air temperatures result in increasing DPLRs.

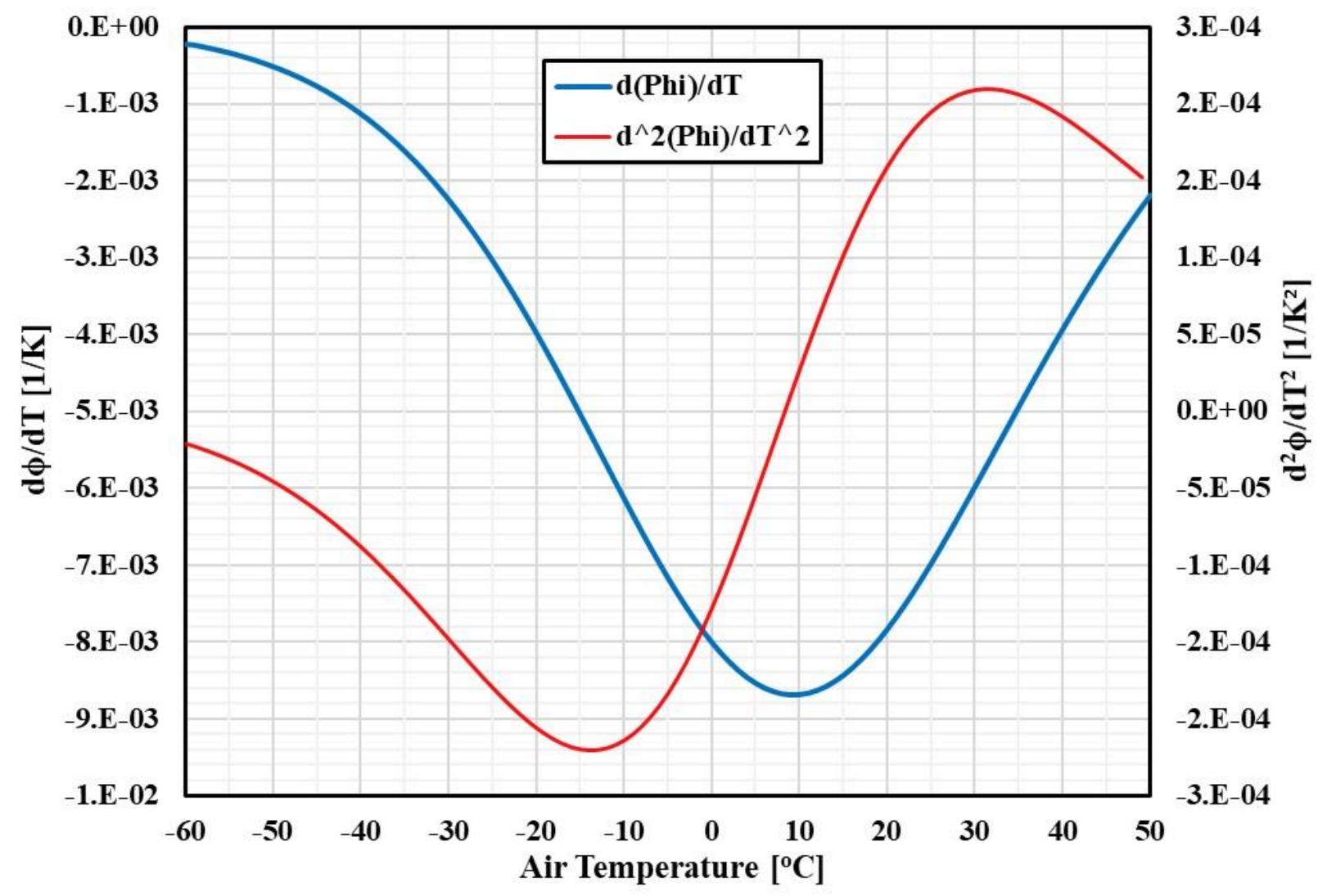

Figure 4: First (blue) and second (red) derivatives of SALR with respect to air temperature.

Other LRs, such as DALR, which is actually a pseudo-constant on Earth, are sketched in Fig. 2. MALR is differing only by small amount from DALR. Hence, it is not particularly interesting for DALR and MALR to deserve a separate plot. On the other hand, ISA LRs are agreed constant standard values based on the altitude zones in terrestrial atmosphere. Once MALR and DPLR are known, the LCL LR is MALR-DPLR difference, i.e., the rate at which the humid air parcels approach saturation defining LCL. This is elementary yet essential process in cloud physics of terrestrial atmosphere. More on LCL models and computations will be presented in upcoming publication. 


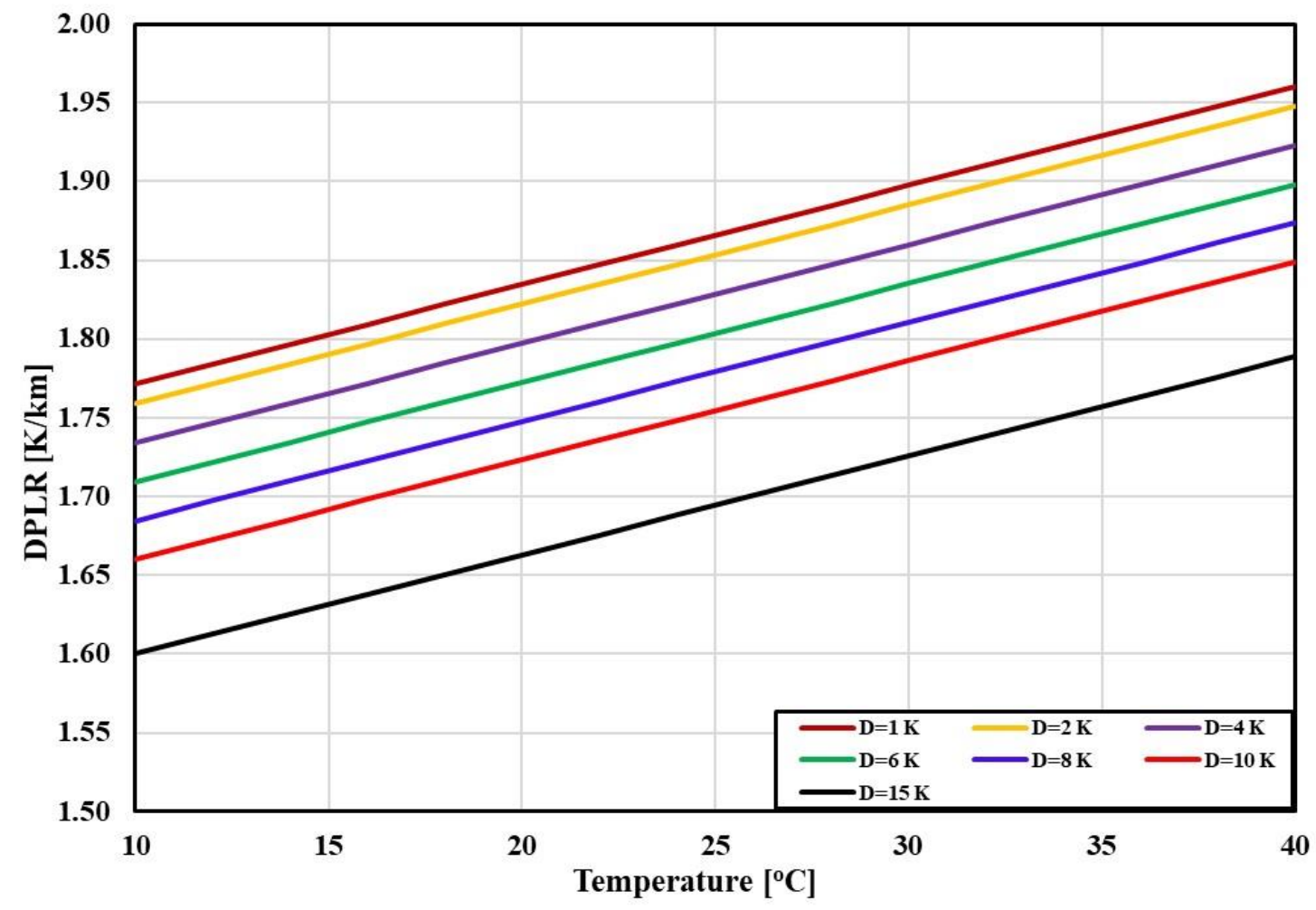

Figure 5: DPLR as a function of air temperature for constant DP depressions.

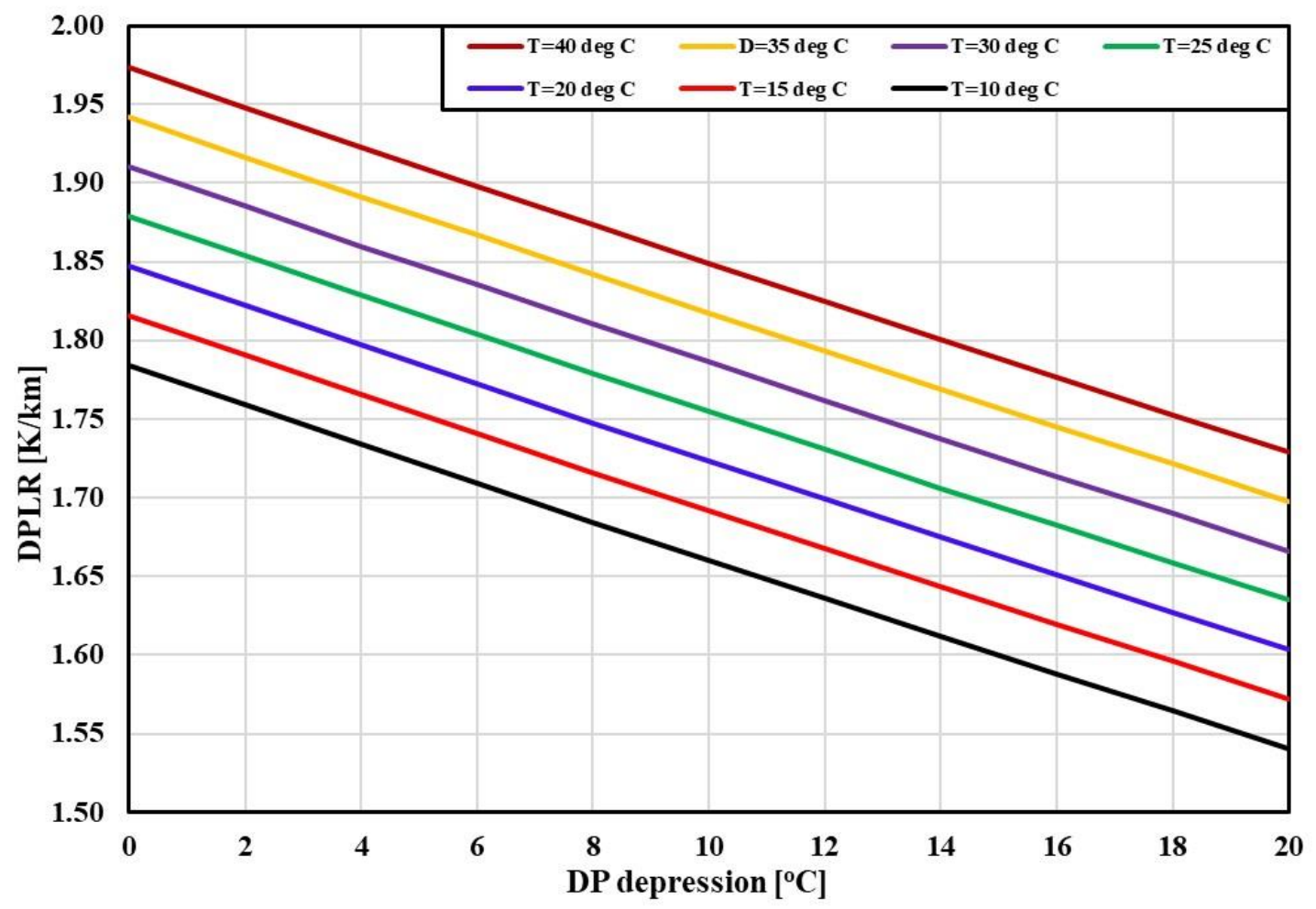

Figure 6: DPLR as a function of DP depression for constant air temperature curves. 


\section{Conclusions}

We have derived and summarized important terrestrial atmosphere temperature lapse rates. The analysis presented here can be applied to other planetary and satellite atmospheres. ALRs, largely, govern vertical atmospheric air stability and creation of some cloud types. To the best of our knowledge, the sensitivity analysis of various atmospheric lapse rates and their dependence on actual ideal-gas air properties and terrestrial gravitational attraction was conducted. SALR, which has DALR as the upper asymptote, showed steepest change at around $9^{\circ} \mathrm{C}$ then flattening out and apparently approaching lower asymptotic solution which has not been investigated as it falls outside of the terrestrial air temperature range. We have analyzed the dependence of DPLR on air temperatures and moisture content. Understanding thermodynamics and dynamics of atmospheric moist air and phase transitions is of fundamental importance to safety and economy of flight operations and aircraft performance.

\section{Author Bio}

Dr. Nihad E. Daidzic is president and chief engineer of AAR Aerospace Consulting, L.L.C. He is also a full professor of Aviation, adjunct professor of Mechanical Engineering, and research graduate faculty at Minnesota State University, Mankato campus. His Ph.D. (Dr.-Ing.) is in fluid mechanics and Sc.D. in mechanical engineering. He was formerly a staff scientist at the National Center for Microgravity Research and the National Center for Space Exploration and Research at NASA Glenn Research Center in Cleveland, $\mathrm{OH}$. He also held various faculty appointments at Vanderbilt University, University of Kansas, and Kent State University. His current research interest is in theoretical, experimental, and computational fluid dynamics, micro- and nano-fluidics, aircraft stability, control, and performance, mechanics of flight, piloting techniques, and aerospace propulsion. Dr. Daidzic is an ATP AMEL and FAA "Gold Seal" CFIA/CFI-IA/ME-IA/CFI-RH/CFI-G/AGI/IGI instructor for several decades with flight experience in airplanes, helicopters, and gliders. 


\section{Nomenclature}

\section{Greek}

$\gamma[-]$

$\varphi[-]$

$\rho\left[\mathrm{kg} / \mathrm{m}^{3}\right]$

$\Gamma[\mathrm{K} / \mathrm{m}]$

Alphabetic

$c[\mathrm{~kJ} / \mathrm{kg} \mathrm{K}] \quad$ Specific heat capacity.

$e[\mathrm{~Pa}]$

$g\left[\mathrm{~m} / \mathrm{s}^{2}\right]$

$h[\mathrm{~kJ} / \mathrm{kg}]$

$l[\mathrm{~J} / \mathrm{kg}]$

$p[\mathrm{~Pa}]$

$\mathrm{q}[-]$

$r[-]$

$z[\mathrm{~m}]$

$D[\mathrm{~K}]$

$H[\mathrm{~m}]$

$L[\mathrm{~J}]$

$M[\mathrm{~kg} / \mathrm{kg}-\mathrm{mol}]$

$R_{0}[\mathrm{~m}]$

$R[\mathrm{~J} / \mathrm{kg} \mathrm{K}]$

$T[\mathrm{~K}]$

$V\left[\mathrm{~m}^{3}\right]$

$W[\mathrm{~J}]$

\section{Subscripts}

\section{$d$ \\ $m$}

$p$
Isentropic coefficient.

Relative humidity.

Density (mass).

Lapse rate.
Water vapor pressure.
Terrestrial gravitational acceleration.

Enthalpy.

Mass specific latent heat (vaporization, fusion, etc.).

Pressure (thermodynamic).

Specific humidity.

Mixture ratio.

Height (Orthometric).

Dew point depression (spread).

Height (Geopotential).

Latent heat (vaporization, fusion, etc.).

Molar Mass.

Radius (average) of spherical Earth approximation.

Gas constant (gas specific).

Temperature.

Volume.

Work (thermodynamic or technical). 
$s$

$v$

$v$

$D P$

$M$

$T P$

\section{Abbreviations}

$\begin{array}{ll}\text { ALR } & \text { Atmospheric Lapse Rate. } \\ \text { CB } & \text { Cumulonimbus cloud (T-Storm). } \\ \text { CN } & \text { Condensation Nuclei. } \\ \text { D } & \text { DP-depression (or spread). } \\ \text { DB } & \text { Dry Bulb } \\ \text { DALR } & \text { Dry (air) Adiabatic Lapse Rate. } \\ \text { DP } & \text { Dew Point. } \\ \text { DPLR } & \text { Dew Point Lapse Rate. } \\ \text { ELR } & \text { Environmental Lapse Rate. } \\ \text { FP } & \text { Frost Point. } \\ \text { GP } & \text { Geopotential } \\ \text { ISALR } & \text { International Standard Atmosphere Lapse Rate. } \\ \text { LCL } & \text { Lifting Condensation Level. } \\ \text { LR } & \text { Lapse Rate. } \\ \text { MALR } & \text { Moist (unsaturated air) Adiabatic Lapse Rate. } \\ \text { RH } & \text { Relative humidity. } \\ \text { SALR } & \text { Saturated (air) Adiabatic Lapse Rate. } \\ \text { WB } & \text { Wet Bulb. }\end{array}$

Saturated.

Vapor, wet.

Constant volume process.

Dew point.

Moist (humid).

Triple point. 


\section{References}

Ambaum, M. H. P. (2008). General relationships between pressure, weight and mass of a hydrostatic fluid. Proceedings of the Royal Society A, 464, 943-950. DOI: 10.1098/rspa.2007.0148.

Berberan-Santos, M. N., Bodunov, E. N., \& Pogliani L. (1997). On the barometric formula. American Journal of Physics, 65(5), 404-412. DOI: 10.1119/1.18555.

Bradbury, T. (2000). Meteorology and Flight: A pilot's guide to weather (3rd Ed.). London, UK: A \& C Black.

Cosgrove, B. (1999). Pilot's Weather: A commonsense approach to meteorology. Vergennes, VT: Plymouth Press.

Daidzic, N. E. (2015a). Efficient general computational method for estimation of standard atmosphere parameters. International Journal of Aviation Aeronautics and Aerospace, 2(1), pp. 1-37. DOI: 10.15394/ijaaa.2015.1053.

Daidzic, N. E. (2015b). Global optimized isothermal and nonlinear models of earth's standard atmosphere. International Journal of Aviation Aeronautics and Aerospace, 2(3), pp. 1-43. DOI: 10.15394/ijaaa.2015.1064.

Daidzic, N. E. (2019). On moist air and dew points. International Journal of Aviation Aeronautics and Aerospace, 6(3), pp. 1-35. DOI: 10.15394/ijaaa.2019.1339.

Dutton, J. A. (2002). The ceaseless wind: An introduction to the theory of atmospheric motion. Mineola, NY: Dover.

Federal Aviation Administration (2016). Aviation weather (AC 00-6B). Department of Transportation (DOT), Washington, DC: Author.

Fleagle, R. G., \& Businger, J. A. (1980). An introduction to atmospheric physics (2nd ed.). New York, NY: Academic Press.

Forrester, F. H. (1981). 1001 questions answered about the weather. New York, NY: Dover.

Houghton, J. (2002). The physics of atmosphere (3rd ed.). Cambridge, United Kingdom: University Press.

International Organization for Standardization (1975). Standard Atmosphere (ISO 2533:1975/Add. 2: 1997(en)). Geneva, Switzerland: Author.

International Civil Aviation Organization (1993). Manual of the ICAO standard atmosphere (Doc 7488-CD, 3rd ed.). Montreal, Canada: Author. 
Iribarne, J. V., \& Cho, H.-R. (1980). Atmospheric physics. Dordrecht, Holland: D. Reidel Publishing Company.

Iribarne, J. V., \& Godson, W. L. (1981). Atmospheric thermodynamics (2nd ed.). Dordrecht, Holland: D. Reidel Publishing Company.

Jeppesen (2015). Guided flight discovery: Instrument Commercial. Englewood, CO: Author.

Lawrence, M. G. (2005). The relationship between relative humidity and the dewpoint temperature in moist air: A simple conversion and applications. Bulletin of the American Meteorological Society (BAMS), 86, 225-233, DOI: 10.1175/BAMS-86-2-225.

Lester, P. (2007). Aviation weather (3rd ed.). Englewood, CO: Jeppesen-Sanderson.

Lowrie, W. (2007). Fundamentals of geophysics (2nd ed.). Cambridge, UK: Cambridge University Press.

NOAA (1976). U.S. standard atmosphere, 1976 (NOAA-S/T 76-1562). Washington, D.C.: U.S. Government Printing Office.

Piggot, D. (1996). Understanding flying weather (2nd ed.). London, UK: A \& C Black.

Reichmann, H. (1993). Cross-country soaring (7th ed.). Translated from German original: Streckensegelflug. Stuttgart, Germany: Motorbuchverlag (English translation by the Soaring Society of America (SSA).

Rogers, R. R. (1979). A short course in cloud physics (2nd ed.). Oxford, UK: Pergamon Press.

Saucier, W. J. (1989). Principles of meteorological analysis. Mineola, NY: Dover.

Stacey, F. D., \& Davis, P. M. (2008). Physics of the earth (4th ed.). Cambridge, UK: Cambridge University Press.

Stull, R. (2016). Practical meteorology: An algebra-based survey of atmospheric science. http://www.eos.ubc.ca/books/Practical_Meteorology/

Torge, W. (2001). Geodesy (3rd ed.). Berlin, Germany: Walter de Gruyter, GmbH.

Tsonis, A. A. (2007). An introduction to atmospheric thermodynamics (2nd ed.). Cambridge, UK: University Press.

Tufty, B. (1987). 1001 questions answered about hurricanes, tornadoes and other natural disasters. New York, NY: Dover.

Wallace, J. M., \& Hobbs, P. V. (2006). Atmospheric science: An introductory survey (2nd ed.). Burlington, MA: Academic Press. 